\title{
Balance Differences between North and South American Older Adults: A Cross-Sectional, Age and Sex Matched Study
}

\author{
Matheus Souza ${ }^{1}$, Daniel Goble ${ }^{2}$, Paige Arney ${ }^{3}$, Edgar Vieira ${ }^{4}$, Gabriela Silveira-Nunes ${ }^{5}$, Leonardo Intelangelo ${ }^{6}$ \\ Michelle Barbosa ${ }^{7}$, and Alexandre Barbosa $7, *$ \\ 1 Department of Physical Therapy, Musculoskeletal Research Group - NIME, Federal University of Juiz de \\ Fora, Brazil; malmeida_1812@hotmail.com \\ 2 Department of Exercise Science, Oakland University, USA; dgoble@oakland.edu \\ 3 Department of Exercise Science, Oakland University, USA; pmarney@oakland.edu \\ 4 Department of Physical Therapy, Florida International University, USA; evieira@fiu.edu \\ 5 Department of Medicine, Musculoskeletal Research Group - NIME, Federal University of Juiz de Fora, \\ Brazil; gabriela.abreu@ufjf.edu.br \\ 6 Department of Physical Therapy, Musculoskeletal Research Unit - UIM, University Center for Assis-tance, \\ Teaching and Research - CUADI, Universidad del Gran Rosario - UGR, Rosario, Argentina, \\ leonardo.intelangelo@gmail.com \\ 7 Department of Physical Therapy, Musculoskeletal Research Group - NIME, Federal University of Juiz de \\ Fora, Brazil; alexandre.barbosa@ufjf.edu.br \\ * Correspondence: alexandre.barbosa@ufjf.edu.br; Tel.: +55 339 9154-1851
}

\begin{abstract}
This study aimed to characterize the risk of falling in low, moderate and high risk participants from two different geographical locations using a portable force-plate. A sample of 390 older adults from South and North America were matched for age, sex, height and weight. All participants performed a standardized balance assessment using a force plate. Participants were classified in low, moderate and high risk of falling. No differences were observed between South and North American men, nor comparing North American men and women. South American women showed the significantly shorter center of pressure path length compared to other groups. The majority of the sample was categorized as having low risk of falling (male: $65.69 \%$ and female: $61.87 \%$ ), with no differences between men and women. Also, no differences were found between North vs. South Americans, nor for falls risk levels when male and female groups were compared separately. In conclusion, South American women had better balance compatible with the status of the 50-59 years' normative age-range. The prevalence of low falls risk was $\sim 61-65 \%$ and the prevalence of moderate to high risk was 16-19 \%. The frequency of fall risk did not differ significantly between North and South Americans, nor between males and females.
\end{abstract}

Keywords: Aging; Accidental Falls; Frail Elderly; Postural Balance

\section{Introduction}

Worldwide expansion of the older adult population is a well-established trend. [1,2] Aging is associated with declines in neuromuscular function and physical performance, $[3,4]$ increased risk of falling, hospitalization, fractures and mortality. $[3,5,6]$ The annual prevalence of falls among older adults in the two largest South American countries has been estimated to be $27 \%$ (Brazil) and 28.5\% (Argentina), respectively. This is similar to the rate seen in the United States (28.7\%). [7,8] The personal and economic burden of falling is significant, representing a total cost of billions of dollars to the public healthcare systems. $[9,10]$ Examining risk factors for falls, such as impaired balance may help identify those at risk and alleviate the physical and psychological impacts of falls.

Balance depends on the ability to maintain the center of mass over one's base of support and is achieved via coordination of complex sensory, motor, and biomechanical 
processes. [11,12] For older adults, balance is especially important in order to recover from disturbances and prevent falls. [13-15] Poor balance is associated with aging-related physical decline, $[16,17]$ with impaired balance variables being correlated to increased falls risk. [18,19] Poor balance and impaired mobility are associated with a $33 \%$ increased risk of recurrent falls in older adults. [20]

Balance can be accurately and reliably measured using a force plate device. [21,22] Force plates measure postural sway based on center of pressure (COP) oscillations, which is equal to the weighted average of forces created when people stand on the force plate for a previously set amount of time. $[23,24]$ Older adults who fall repeatedly have a greater magnitude COP oscillations than those without a history of falling. [25,26] Furthermore, older people with increased postural sway present a greater likelihood of falling. $[21,25,26]$

Force plate-based balance assessments in older adults have been somewhat limited due to cost $(\$ 10,000-\$ 100,000)$, technical difficulties, and time/training to process and extract data from the raw signals. $[27,28]$ Force plates often lack user-friendly software that are time efficient, reliable, and intuitive. [29] However, recent developments led to reliable and low-cost force plates with user-friendly software. [30] The Balance Tracking System (BTrackS) is a low-cost $(<\$ 2,000)$ and portable $(<7 \mathrm{~kg}$, USB-powered) force plate (FDA class 1 registered device) with user-friendly software for balance assessment that have been shown to be valid and reliable for testing community dwelling older adults. [31] BTrackS has also been shown to accurately detect the risk of falling in older adults based on their balance indicators. [29]

The largest known normative database of older adult balance was created using the BTrackS balance and fall risk test protocol. [32] This protocol measures the postural sway of an individual while standing still with eyes closed for three trials of 20 -seconds. The numerical results are converted in a categorical model according to a predefined range of body sway, allowing early detection of balance impairments. [29,31] According to a manufacturer report (https://balancetrackingsystems.com/wpcontent/uploads/2019/05/Validating-BTrackS-FRA.pdf), the low-risk category equates to a $29 \%$ likelihood of falling in the next 12 months. This likelihood rises to $42 \%$ for those in the moderate category, and to $51 \%$ for those classified in the high risk category. [29]

One limitation of the BTrackS system is that the normative data utilized is almost exclusively based on values from the United States population. Considering the lack of studies comparing balance data in different locations, the primary aim of the present study was to compare the balance of a representative sample of healthy North and South American older adults by sex. Additionally, this study aimed to characterize the risk of falling in low, moderate and high risk categories using BTrackS criteria. It was hypothesized that balance and falls risk would not be significantly different between north and south America older adults, nor between older men and women.

\section{Materials and Methods}

\subsection{Participants}

The present study was a cross-sectional secondary analysis of the balance data collected from previous studies with older adults using the same equipment and procedure for all participants. A total of 390 older adults ( $\geq 60$ years of age) of both sexes were included (Table 1). Participants were taken from two geographical locations (North vs South America) and closely matched for age, sex, height and weight. Specifically, the participants were selected from three of the largest countries on the South and North American continents: Brazil and Argentina (South America), as well as the United States (North America). Four comparison groups were therefore determined from the sample including: South American Men, North American Men, South American Women, and North American Women. 
All procedures were performed on-site without any prior practice. Attendance was taken as compliance with the assessment protocol. No co-interventions were performed in either group, and no adverse effects were reported by any participant during any procedure. Prior to testing, the participants were familiarized with all physical assessment procedures. The sample was physically independent (level 3 or 4 on Functional Status), [33] and cognitively able to understand the procedures (score $>21$ on the Mini-Mental State Examination for people with low education) [34]. Exclusion criteria were incidence of cardiovascular disease, unstable proliferative retinopathy, end-stage renal disease, uncontrolled hypertension [35]. All participants were free of any knee or hip injury which could affect their balance. The participants were also cleared from medication that could cause dizziness as a side effect.

Based on a post hoc power calculation an obtained effect size $\left(\varepsilon^{2}\right)$ of 0.252 , with an alpha level of 5\%, was determined for the total sample size of 390 , and an actual power of 0.992 was determined for the four individual groups. The sample power was calculated using G-POWER ${ }^{\mathrm{TM}}$ software (Version 3.1.5, Franz Faul, Universitat Kiel, Germany). Procedures for this study were approved by the institutional review boards from all countries involved (Argentinian protocol number 38/16 UGR-Rosario; Brazilian protocol number 59862316.7.0000.5147; USA protocol number 2203100).

Table 1. Participants' characteristics in median (minimum-maximum). Mann-Whitney non-parametric test results.

\begin{tabular}{ccccccc}
\hline & \multicolumn{3}{c}{ Men } & \multicolumn{3}{c}{ Women } \\
Outcome & South & North & $p$ & South & North & $p$ \\
\hline $\mathbf{n}$ & 44 & 44 & - & 151 & 151 & - \\
Age (years) & $70(61-86)$ & $71(61-87)$ & 0.89 & $70(60-98)$ & $71(60-99)$ & 0.95 \\
Height (cm) & $167(105-184)$ & $168(155-185)$ & 0.87 & $155(137-176)$ & $155(124-175)$ & 0.56 \\
Weight (kg) & $74.5(52-94)$ & $76.5(50-98)$ & 0.59 & $64(42-98)$ & $64(43-127)$ & 0.94 \\
\hline
\end{tabular}

\subsection{Equipment and procedure}

The BTrackS Balance Plate (Balance Tracking System, San Diego, CA, USA) was used to assess balance. This device consists of a force platform $(40 \times 60 \mathrm{~cm}$, sampling frequency of $25 \mathrm{~Hz}$ ) with four implanted strain gauges that determine the center-of-pression (COP) excursion distance when stood upon. The BTrackS sampling frequency satisfied the Nyquist theorem for the slow $(<10 \mathrm{~Hz})$ COP changes measured in the present study. Previous work has shown BTrackS to perform with the same accuracy/precision as a laboratory-grade force platform [30]. Prior to testing, the force platform was leveled via adjustable legs, and connected to the computer through a USB cable which also provided power to the plate.

The procedure for testing required all participants to perform four, 20s two-legged stance trials (figure 1). The first trial was for familiarization and was discarded before analysis. The remaining three, non-familiarization trials were used to determine the result. Trials were conducted in a closed room to reduce noise and disturbances. Participants were instructed to firstly look straight ahead and to stand as still as possible on the BTrackS Balance Plate with eyes closed, hands on hips and feet shoulder width apart. Participants were monitored by the tester during all trials to avoid a fall episode. 


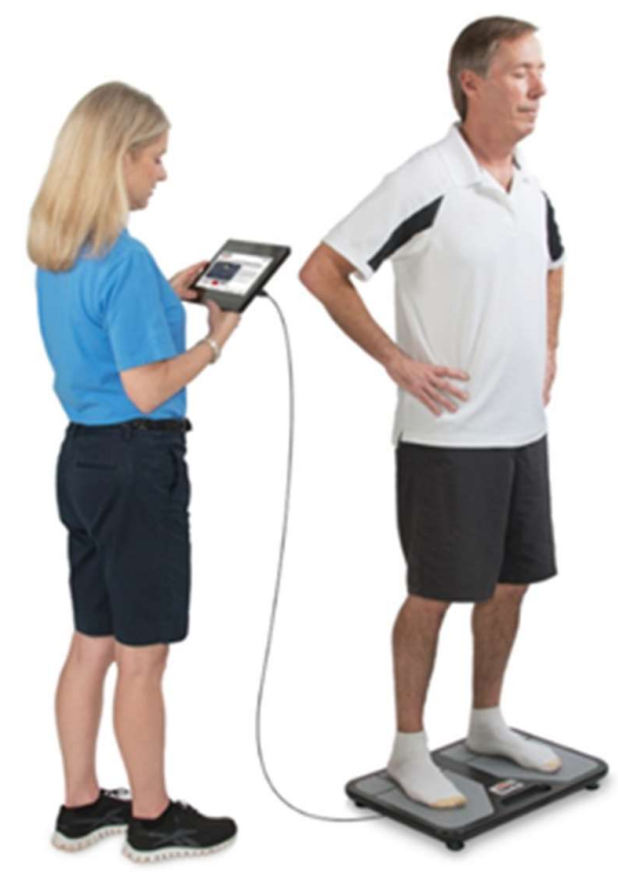

Figure 1. BTracks testing setup for a fall risk assessment.

The result for each test was calculated by the BTrackS Assess Balance software, equivalent to the average total COP path length in centimeters $(\mathrm{cm})$ across accountable trials. COP path length is a proxy for postural sway magnitude where larger BBT values are indicative of greater postural sway $[21,32,36]$. Path length was determined by first quantifying the distance between successive registered COP locations according to the following formula:

$$
\text { distance }=\left(\left[\mathrm{COP}_{\mathrm{x} 2}-\mathrm{COP}_{\mathrm{x} 1}\right]^{2}+\left[\mathrm{COP}_{\mathrm{y} 2}-\mathrm{COP}_{\mathrm{y} 1}\right]^{2}\right)^{0.5}
$$

Where $\mathrm{COP}_{\mathrm{x} 2}$ and $\mathrm{COP}_{\mathrm{x} 1}$ are adjacent time-points in the $\mathrm{COP}_{\mathrm{x}}$ (medial/lateral) time series, and $\mathrm{COP}_{\mathrm{y} 2}$ and $\mathrm{COP}_{\mathrm{y} 1}$ are adjacent time-points in the $\mathrm{COP}_{\mathrm{y}}$ (anterior/posterior) time series. The sum of all distances was then added together to obtain the total path length. Participants were also classified into one of three fall risk categories: low risk (postural sway range: 0-32 cm for men and 0-30 cm for women); moderate risk (postural sway range: $33-$ $40 \mathrm{~cm}$ for men and 31-38 cm for women); and high risk (postural sway range: $41+$ for men and 39+ for women). [27]

\subsection{Statistical analysis}

Shapiro-Wilk and the Levene's tests were first performed to assess the normality and equality of variances for each group tested. Given that normality was not achieved, descriptives for this study are presented as median, minimum and maximum values. For statistical comparisons of group data, non-parametric Kruskall Wallis tests were used with Dwass-Steel-Critchlow-Fligner post hoc correction protect against multiple comparisons. The between-group fall risk categorization frequencies (low, moderate and high risk) were evaluated using a chi-square test for ordinal comparisons. Significance was set at the $p<0.05$ level for all tests. Standardized differences for comparisons were analyzed using the rank biserial correlation effect size (ES). The magnitude of the ES was qualitatively interpreted using the following thresholds: 0.01-0.19; small: 0.20-0.49; moderate: 0.50-0.79; large: 0.81.19; very large: 1.2-1.99; and huge: $>2$ [37]. All analysis was performed using the JAMOVI software (version 1.6.15.0, 2021; retrieved from https://www.jamovi.org). 


\section{Results}

No differences were observed between men (South American men: median $=32 \mathrm{~cm}$ [minimum-maximum: 16-75 cm], Northern American men: $33 \mathrm{~cm}$ [16-79 cm]; $p=0.99$; ES=0.001), nor comparing North American men and women (North American women: 27 $\mathrm{cm}[10-115 \mathrm{~cm}] ; p=0.14 ; \mathrm{ES}=0.21)$. However, South American women showed the lowest path length compared to other groups (South American women: $24 \mathrm{~cm}$ [10-70 cm]; all $p<0.05$ ), with effect sizes ranging from small to moderate (figure 2 ).

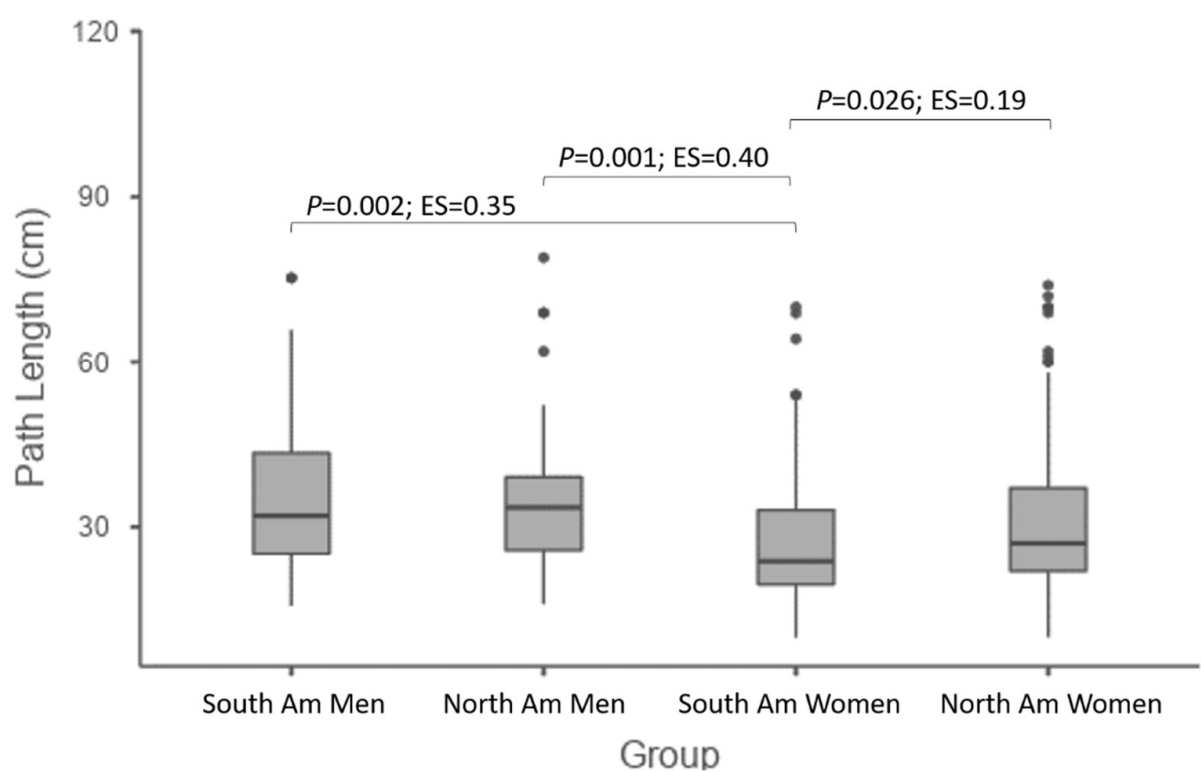

Figure 2. Box plot of pairwise comparisons. Box trace = median values; box limits = minimum-maximum; vertical trace = standard deviation; dots = outliers; ES = effect size. Significant differences assigned.

Considering the overall sample, the majority of the sample was categorized as low risk of falling (male: $65.69 \%$ and female: $61.87 \%$ ). The frequency of the low risk category was higher $\left(\chi^{2}=8.32, p=0.016\right)$ compared to moderate (men: $16.34 \%$ and women: 18.18 $\%$ ) and high risks (male: $17.97 \%$ and female: $19.95 \%$ ). No differences were observed comparing men vs. women. Also, no differences were found between North vs. South America (table 2), nor for categories' frequencies when male or female groups were compared separately.

Table 2. Risk of falling frequencies in \% of the sample per gender.

\begin{tabular}{|c|c|c|c|c|c|}
\hline \multirow{2}{*}{ Gender } & \multirow{2}{*}{ Group } & \multicolumn{3}{|c|}{ Risk of Falling } & \multirow{2}{*}{ Difference } \\
\hline & & Low & Moderate & High & \\
\hline \multirow{2}{*}{ Male } & South & $26.67 \%$ & $6.67 \%$ & $16.67 \%$ & \multirow{2}{*}{$\chi^{2}=4.40, p=0.111$} \\
\hline & North & $23.33 \%$ & $15.56 \%$ & $11.11 \%$ & \\
\hline \multirow{2}{*}{ Female } & South & $34.64 \%$ & $8.50 \%$ & $6.86 \%$ & \multirow{2}{*}{$\chi^{2}=3.81, p=0.149$} \\
\hline & North & $30.72 \%$ & $8.17 \%$ & $11.11 \%$ & \\
\hline
\end{tabular}

\section{Discussion}

The primary focus of the present study was to compare the balance of North vs. South American older adults grouped by sex using the same device (i.e. BTrackS). Only South 
American women showed differences compared to other groups, with improved postural control (i.e. better balance) at a small-to-moderate effect size. There were no differences seen in the probability of fall risk across sex and geographic region. To the best of our knowledge, this is the first study comparing the balance of a representative sample of older adults from distinct continents using a standardized protocol with the same device. These results suggest that balance ability may differ to some extent across large geographical areas. In this case, pre-existing balance norms would require some adjustment based on geographic location and sex demographics.

In contrast to our results, one previous study showed decreased balance control in older women compared to men. [38] The authors also reported that older women had greater COP displacement but less sway velocity than their male counterpart, especially in mediolateral direction. However, the protocol used to assess the balance included only 2 trials with open eyes, $30 \mathrm{~s}$ of data collection and $5 \mathrm{~min}$ of rest between trials. The present protocol was performed with closed eyes as the vision is a major contributor to postural stability. [39] The visual system inputs information on head position relative to the surrounding environment, which might be used in a feedforward fashion to anticipate a loss in balance. [40] On the other hand, the closed eyes task imposes greater efforts to proprioceptive system, becoming the main source of neural feedback during an imbalance situation. [13,40,41] Further, the current protocol was extensively validated in thousands of subjects, with consistent results showing greater postural sway in older men compared to age-matched older women. [21,30,40,42] One of these previous studies compared the balance results of 812 older men compared to 2,341 older women, with a mean difference of $\sim 8 \mathrm{~cm}$ on the path length variable. [21] The difference was consistent across all 3 ranges of older age (60-70, 70-80, and 80+ years).

The male groups' overall results along with the North American women are in accordance to the expected for their age. [21] The South American women, however, showed a body sway compatible with younger normative data ( 50-59 years). Due to the study design, no cause-effect can be inferred from the present results. Nevertheless, it is well known that some factors may influence the balance outcomes in older adults. According to an updated meta-analysis with 19,478 participants, the greater effects were seen from exercise protocols that challenged balance and involved more than 3 hours/week of exercise. [43-45] Thus, a possible explanation for the present results could be the South American women's engagement in physical activities impacting the musculoskeletal development and the consequent balance improvements. Another possible explanation for the present findings is the possible higher rate of falls and frailty in North American older adults (15\%) compared to South American older adults (9\%). [46,47] Impaired balance is also predictive of bad outcomes in older adults, such as frailty and higher prevalence of falls. $[14,48,49]$ Considering the overall prevalence of falls, South American older adults have an average rate of $27 \%$ while North American older adults have a higher rate of 28.7 $\%$ (33.9\% in certain states). [8,50,51]

A secondary aim of the present study was to examine the frequency of low, moderate and high risk of falling. Despite the higher percentage of overall older people under low risk of falling independent of location, an important proportion of participants was classified as moderate or high risk of falling ( $35 \%)$. Balance is maintained or restored in the hip strategy mainly by movement of the body around the hip and trunk, while in the ankle strategy it is restored mainly by the movement of the body around the ankle as a single inverted pendulum with minimal movement about superior joints. [52,53] In an unexpected situation of imbalance, an older adult at high risk of falling tends to use the hip strategy to maintain postural stability compared to another under low risk of falling. [54] The use of such strategy may increase the confidence, as the COP tends to move faster to stay within the base of support. [54] Mechanically, the foot's ability to exert torque in 
contact with the support surface limits the ankle strategy, as distal muscles are acting far from the body's COP to stabilize the full body sway. [53] Although both strategies are biomechanically different, they have the main goal to maintain the forward progression and the upright balance.

As individuals in the moderate and high risk category are recommended to be targeted for balance intervention programs, [29] health care professionals may benefit their older patients with early diagnostics and active intervention to prevent bad outcomes that follows an episode of fall (e.g. head trauma, spine and hip fractures, hospitalization). $[55,56]$ Also, a comprehensive balance screening focused on the lower limb and trunk's muscles is essential to target the segments in need of care, as the current protocol is performed using the ankle strategy. Thus, the classification is also based on the level of COP displacement considering only this strategy.

An important limitation of the present study is the cross-sectional design, which does not allow cause-effect inferences. Further investigation is needed to determine changes over time in individuals receiving interventions to improve the balance status. It is also important to address that the present study only assessed the postural balance, one aspect of the functionality. The level of physical function of the sample was also a limitation. Although the falling status was properly categorized between groups, all participants were at a high functioning level. Institutionalized older women or those presenting high levels of frailty may show distinct balance patterns.

\section{Conclusions}

South and North American men have the same balance status, as well as the North American women. On the other hand, South American women showed improved balance compatible with the status of the previous normative age-range (50-59 years). A higher percentage of overall older people under low risk of falling was observed independent of location, but an important proportion of participants was classified as moderate or high risk of falling. The balance categories frequencies did not vary considering the location (South vs. North), nor considering the gender factor.

Author Contributions: Conceptualization, A.B. and D.G.; methodology, A.B.; software, A.B. and M.S.; validation, M.A., M.B. and G.S.; formal analysis, A.B., D.G. and E.V.; investigation, M.A., P.A. and L.I.; resources, A.B., L.I. and D.G.; data curation, P.A., D.G. and A.B.; writing-original draft preparation, A.B., E.V. and D.G.; writing-review and editing, D.G., E.V. A.B. and P.A.; visualization, M.A and M.B.; supervision, A.B. and D.G.; project administration, A.B and D.G.; funding acquisition, D.G. and A.B. All authors have read and agreed to the published version of the manuscript.

Funding: This study was supported in part by the Coordenação de Aperfeiçoamento de Pessoal de Nível Superior - Brasil (CAPES) - Finance Code 001 and by the Fundação de Amparo à Pesquisa de Minas Gerais (FAPEMIG). This research was funded by the Federal University of Juiz de Fora, supporting the APC.

Institutional Review Board Statement: The study was conducted according to the guidelines of the Declaration of Helsinki, and approved by the Ethics Committee of the Federal University of Juiz de Fora - Brazil (protocol number 59862316.7.0000.5147); University of Gran Rosario - Argentina (protocol number 38/16); Oakland University - USA (protocol number 2203100).

Informed Consent Statement: Informed consent was obtained from all subjects involved in the study.

Data Availability Statement: Raw data is available from the following doi: 10.17632/cyg8vcxytn.1.

Acknowledgments: Special thanks to UFJF-GV Department of Physical Therapy and the staff of the NIME/UFJF-GV. 
Conflicts of Interest: DG receives royalties from a patent (US Patent 10,660,558) related to the balance technology in this study. In addition, he has an equity stake (i.e. stock options) and performs contract work for Balance Tracking Systems Inc., the parent company of the BTrackS Balance Plate and BTrackS Assess Balance Advance software. This conflict is mitigated by a management plan put in place by his academic institution (Oakland University) to ensure the integrity of his research. The other authors of this work have no conflicts of interest to declare.

\section{References}

1. Raj, I.S.; Bird, S.R.; Shield, A.J. Aging and the force-velocity relationship of muscles. Exp. Gerontol. 2010, 45, 8190, doi:10.1016/j.exger.2009.10.013.

2. World Population Ageing 2019 Highlights; UN, 2019; ISBN 9789210045537.

3. Yeung, S.S.Y.; Reijnierse, E.M.; Pham, V.K.; Trappenburg, M.C.; Lim, W.K.; Meskers, C.G.M.; Maier, A.B. Sarcopenia and its association with falls and fractures in older adults: A systematic review and meta-analysis. J. Cachexia. Sarcopenia Muscle 2019, 10, 485-500, doi:10.1002/jcsm.12411.

4. Dent, E.; Morley, J.E.; Cruz-Jentoft, A.J.; Woodhouse, L.; Rodríguez-Mañas, L.; Fried, L.P.; Woo, J.; Aprahamian, I.; Sanford, A.; Lundy, J.; et al. Physical Frailty: ICFSR International Clinical Practice Guidelines for Identification and Management. J. Nutr. Heal. Aging 2019, 23, 771-787, doi:10.1007/s12603-019-1273-z.

5. Trombetti, A.; Reid, K.F.; Hars, M.; Herrmann, F.R.; Pasha, E.; Phillips, E.M.; Fielding, R.A. Age-associated declines in muscle mass, strength, power, and physical performance: impact on fear of falling and quality of life. Osteoporos. Int. 2016, 27, 463-71, doi:10.1007/s00198-015-3236-5.

6. McKinnon, N.B.; Connelly, D.M.; Rice, C.L.; Hunter, S.W.; Doherty, T.J. Neuromuscular contributions to the agerelated reduction in muscle power: Mechanisms and potential role of high velocity power training. Ageing Res. Rev. 2017, 35, 147-154, doi:10.1016/j.arr.2016.09.003.

7. Chang, V.C.; Do, M.T. Risk Factors for Falls Among Seniors: Implications of Gender. Am. J. Epidemiol. 2015, 181, 521-531, doi:10.1093/aje/kwu268.

8. Bergen, G.; Stevens, M.R.; Burns, E.R. Falls and Fall Injuries Among Adults Aged $\geq 65$ Years - United States, 2014. MMWR. Morb. Mortal. Wkly. Rep. 65, 993-998.

9. Heinrich, S.; Rapp, K.; Rissmann, U.; Becker, C.; König, H.-H. Cost of falls in old age: a systematic review. Osteoporos. Int. 2010, 21, 891-902, doi:10.1007/s00198-009-1100-1.

10. Vieira, E.R.; Palmer, R.C.; Chaves, P.H.M. Prevention of falls in older people living in the community. BMJ 2016, 353, i1419, doi:10.1136/bmj.i1419.

11. Pollock, A.S.; Durward, B.R.; Rowe, P.J.; Paul, J.P. What is balance? Clin. Rehabil. 2000, 14, 402-406, doi:10.1191/0269215500cr342oa.

12. Moral-Munoz, J.A.; Esteban-Moreno, B.; Herrera-Viedma, E.; Cobo, M.J.; Pérez, I.J. Smartphone Applications to Perform Body Balance Assessment: a Standardized Review. J. Med. Syst. 2018, 42, 119, doi:10.1007/s10916-0180970-1. 
13. Shumway-Cook, A.; Woollacott, M. Attentional demands and postural control: the effect of sensory context. J. Gerontol. A. Biol. Sci. Med. Sci. 2000, 55, M10-6.

14. Howcroft, J.; Lemaire, E.D.; Kofman, J.; Mcllroy, W.E. Elderly fall risk prediction using static posturography. PLoS One 2017, 12, 1-13, doi:10.1371/journal.pone.0172398.

15. Amiridis, I.G.; Hatzitaki, V.; Arabatzi, F. Age-induced modifications of static postural control in humans. Neurosci. Lett. 2003, 350, 137-140, doi:10.1016/S0304-3940(03)00878-4.

16. Lesinski, M.; Hortobágyi, T.; Muehlbauer, T.; Gollhofer, A.; Granacher, U. Effects of Balance Training on Balance Performance in Healthy Older Adults: A Systematic Review and Meta-analysis. Sport. Med. 2015, 45, 1721-1738, doi:10.1007/s40279-015-0375-y.

17. Hamed, A.; Bohm, S.; Mersmann, F.; Arampatzis, A. Follow-up efficacy of physical exercise interventions on fall incidence and fall risk in healthy older adults: a systematic review and meta-analysis. Sport. Med. - Open 2018, 4, 56, doi:10.1186/s40798-018-0170-z.

18. Kukidome, D.; Nishikawa, T.; Sato, M.; Nishi, Y.; Shimamura, R.; Kawashima, J.; Shimoda, S.; Mizuta, H.; Araki, E. Impaired balance is related to the progression of diabetic complications in both young and older adults. $J$. Diabetes Complications 2017, 31, 1275-1282, doi:10.1016/j.jdiacomp.2017.05.014.

19. Oliveira, M.R.; Vieira, E.R.; Gil, A.W.O.; Fernandes, K.B.P.; Teixeira, D.C.; Amorim, C.F.; Da Silva, R.A. Onelegged stance sway of older adults with and without falls. PLoS One 2018, 13, doi:10.1371/journal.pone.0203887.

20. Jehu, D.A.; Davis, J.C.; Falck, R.S.; Bennett, K.J.; Tai, D.; Souza, M.F.; Cavalcante, B.R.; Zhao, M.; Liu-Ambrose, T. Risk factors for recurrent falls in older adults: A systematic review with meta-analysis. Maturitas 2021, 144, 23-28, doi:10.1016/j.maturitas.2020.10.021.

21. Goble, D.J.; Baweja, H.S. Postural sway normative data across the adult lifespan: Results from 6280 individuals on the Balance Tracking System balance test. Geriatr. Gerontol. Int. 2018, 1225-1229, doi:10.1111/ggi.13452.

22. Błaszczyk, J.W. The use of force-plate posturography in the assessment of postural instability. Gait Posture 2016, 44, 1-6, doi:10.1016/j.gaitpost.2015.10.014.

23. Melzer, I.; Kurz, I.; Oddsson, L.I.E. A retrospective analysis of balance control parameters in elderly fallers and non-fallers. Clin. Biomech. 2010, 25, 984-988, doi:10.1016/j.clinbiomech.2010.07.007.

24. Melzer, I. Postural stability in the elderly: a comparison between fallers and non-fallers. Age Ageing 2004, 33, 602-607, doi:10.1093/ageing/afh218.

25. Johansson, J.; Jarocka, E.; Westling, G.; Nordström, A.; Nordström, P. Predicting incident falls: Relationship between postural sway and limits of stability in older adults. Hum. Mov. Sci. 2019, 66, 117-123, doi:10.1016/j.humov.2019.04.004.

26. Johansson, J.; Nordström, A.; Gustafson, Y.; Westling, G.; Nordström, P. Increased postural sway during quiet stance as a risk factor for prospective falls in community-dwelling elderly individuals. Age Ageing 2017, 46, 964- 
970, doi:10.1093/ageing/afx083.

27. Goble, D.J.; Baweja, N.; Baweja, H.S. BTrackS. Home Healthc. Now 2019, 37, 355-356, doi:10.1097/NHH.0000000000000823.

28. O'Connor, S.M.; Baweja, H.S.; Goble, D.J.; O'Connor, S.M.; Baweja, H.S.; Goble, D.J. Validating the BTrackS Balance Plate as a low cost alternative for the measurement of sway-induced center of pressure. J. Biomech. 2016, 49, 4142-4145, doi:10.1016/j.jbiomech.2016.10.020.

29. Goble, D.J.; Baweja, N.; Baweja, H.S. BTrackS: A Low-Cost, Portable Force Plate for Objectively Measuring Balance Deficits and Fall Risk. Home Healthc. now 2019, 37, 355-356, doi:10.1097/NHH.0000000000000823.

30. O'Connor, S.M.; Baweja, H.S.; Goble, D.J. Validating the BTrackS Balance Plate as a low cost alternative for the measurement of sway-induced center of pressure. J. Biomech. 2016, 49, 4142-4145, doi:10.1016/j.jbiomech.2016.10.020.

31. Levy, S.S.; Thralls, K.J.; Kviatkovsky, S.A. Validity and Reliability of a Portable Balance Tracking System, BTrackS, in Older Adults. J. Geriatr. Phys. Ther. 2018, 41, 102-107, doi:10.1519/JPT.0000000000000111.

32. Goble, D.J.; Baweja, H.S. Normative Data for the BTrackS Balance Test of Postural Sway: Results from 16,357 Community-Dwelling Individuals Who Were 5 to 100 Years Old. Phys. Ther. 2018, 98, 779-785, doi:10.1093/ptj/pzy062.

33. Snejdrlova, M.; Kalvach, Z.; Topinkova, E.; Vrablik, M.; Prochazkova, R.; Kvasilova, M.; Lanska, V.; Zlatohlavek, L.; Prusikova, M.; Ceska, R. APOE polymorphism as a potential determinant of functional fitness in the elderly regardless of nutritional status. Neuro Endocrinol. Lett. 2011, 32 Suppl 2, 51-4.

34. Folstein, M.F.; Folstein, S.E.; McHugh, P.R. “Mini-mental state." J. Psychiatr. Res. 1975, 12, 189-198, doi:10.1016/0022-3956(75)90026-6.

35. Young, W.R.; Mark Williams, A. How fear of falling can increase fall-risk in older adults: Applying psychological theory to practical observations. Gait Posture 2015, 41, 7-12, doi:10.1016/j.gaitpost.2014.09.006.

36. Goble, D.J.; Hearn, M.C.; Baweja, H.S. Combination of BTrackS and Geri-Fit as a targeted approach for assessing and reducing the postural sway of older adults with high fall risk. Clin. Interv. Aging 2017, 12, 351-357, doi:10.2147/CIA.S131047.

37. Sawilowsky, S.S. New Effect Size Rules of Thumb. J. Mod. Appl. Stat. Methods 2009, 8, 597-599, doi:10.22237/jmasm/1257035100.

38. Kim, J.-W.; Eom, G.-M.; Kim, C.-S.; Kim, D.-H.; Lee, J.-H.; Park, B.K.; Hong, J. Sex differences in the postural sway characteristics of young and elderly subjects during quiet natural standing. Geriatr. Gerontol. Int. 2010, 10, 191-198, doi:10.1111/j.1447-0594.2009.00582.x.

39. Beauchet, O.; Levinoff, E.J;; Allali, G. Decrease in Upright Postural Sway from Open to Closed Eyes: Episodic Memory Impairment Matters, Too. J. Am. Geriatr. Soc. 2016, 64, 1142-1144, doi:10.1111/jgs.14115. 
40. Goble, D.J.; Brar, H.; Brown, E.C.; Marks, C.R.; Baweja, H.S. Normative data for the Balance Tracking System modified Clinical Test of Sensory Integration and Balance protocol. Med. Devices Evid. Res. 2019, Volume 12, 183191, doi:10.2147/MDER.S206530.

41. Chen, E.W.; Fu, A.S.N.; Chan, K.M.; Tsang, W.W.N. Balance control in very old adults with and without visual impairment. Eur. J. Appl. Physiol. 2012, 112, 1631-1636, doi:10.1007/s00421-011-2139-1.

42. Goble, D.J.; Manyak, K.A.; Abdenour, T.E.; Rauh, M.J.; Baweja, H.S. An initial evaluation of the btracks balance plate and sports balance software for concussion diagnosis. Int. J. Sports Phys. Ther. 2016, 11, 149-55.

43. Sherrington, C.; Michaleff, Z.A.; Fairhall, N.; Paul, S.S.; Tiedemann, A.; Whitney, J.; Cumming, R.G.; Herbert, R.D.; Close, J.C.T.; Lord, S.R. Exercise to prevent falls in older adults: an updated systematic review and metaanalysis. Br. J. Sports Med. 2017, 51, 1750-1758, doi:10.1136/bjsports-2016-096547.

44. Morrison, S.; Colberg, S.R.; Parson, H.K.; Vinik, A.I. Relation between risk of falling and postural sway complexity in diabetes. Gait Posture 2012, 35, 662-8, doi:10.1016/j.gaitpost.2011.12.021.

45. Franco, M.R.; Grande, G.H.D.D.; Padulla, S.A.T.T. Effect of pilates exercise for improving balance in older adults (PEDro synthesis). Br. J. Sports Med. 2018, 52, 199-200, doi:10.1136/bjsports-2016-097073.

46. Andrade, J.M.; Duarte, Y.A.D.O.; Alves, L.C.; Andrade, F.C.D.; Souza Junior, P.R. De; Lima-Costa, M.F.; Andrade, F.B. de Frailty profile in Brazilian older adults. Rev. Saude Publica 2019, 52, 17s, doi:10.11606/s15188787.2018052000616.

47. Bandeen-Roche, K.; Seplaki, C.L.; Huang, J.; Buta, B.; Kalyani, R.R.; Varadhan, R.; Xue, Q.-L.; Walston, J.D.; Kasper, J.D. Frailty in Older Adults: A Nationally Representative Profile in the United States. Journals Gerontol. Ser. A Biol. Sci. Med. Sci. 2015, 70, 1427-1434, doi:10.1093/gerona/glv133.

48. Tsai, Y.C.; Hsieh, L.F.; Yang, S. Age-related changes in posture response under a continuous and unexpected perturbation. J. Biomech. 2014, 47, 482-490, doi:10.1016/j.jbiomech.2013.10.047.

49. Ogilvie, M.; Wallen, M.P.; Talpey, S.W. Agile ageing - A modifiable vital sign to mitigate the risk of falls in older adults? Med. Hypotheses 2021, 148, 110517, doi:10.1016/j.mehy.2021.110517.

50. Filho, J.E.; Borel, W.P.; Mata Diz, J.B.; Carvalho Barbosa, A.W.; Britto, R.R.; Felício, D.C. Prevalence of falls and associated factors in community-dwelling older Brazilians: A systematic review and meta-analysis. Cad. Saude Publica 2019, 35, 1-16, doi:10.1590/0102-311X00115718.

51. Moreland, B.; Kakara, R.; Henry, A. Trends in Nonfatal Falls and Fall-Related Injuries Among Adults Aged $\geq 65$ Years - United States, 2012-2018. MMWR. Morb. Mortal. Wkly. Rep. 2020, 69, 875-881, doi:10.15585/mmwr.mm6927a5.

52. Horak, F.B.; Nashner, L.M. Central programming of postural movements: adaptation to altered support-surface configurations. J. Neurophysiol. 1986, 55, 1369-1381, doi:10.1152/jn.1986.55.6.1369.

53. Blenkinsop, G.M.; Pain, M.T.G.; Hiley, M.J. Balance control strategies during perturbed and unperturbed balance 
in standing and handstand. R. Soc. Open Sci. 2017, 4, 161018, doi:10.1098/rsos.161018.

54. Horak, F.B. Postural orientation and equilibrium: what do we need to know about neural control of balance to prevent falls? Age Ageing 2006, 35 Suppl 2, ii7-ii11, doi:10.1093/ageing/afl077.

55. Taylor, C.A.; Bell, J.M.; Breiding, M.J.; Xu, L. Traumatic Brain Injury-Related Emergency Department Visits, Hospitalizations, and Deaths - United States, 2007 and 2013. MMWR. Surveill. Summ. 2017, 66, 1-16, doi:10.15585/mmwr.ss6609a1.

56. Davenport, K.; Alazemi, M.; Sri-On, J.; Liu, S. Missed Opportunities to Diagnose and Intervene in Modifiable Risk Factors for Older Emergency Department Patients Presenting After a Fall. Ann. Emerg. Med. 2020, 76, 730738, doi:10.1016/j.annemergmed.2020.06.020. 